\title{
Nanocrystal Aerogels with Coupled or Decoupled Building Blocks
}

\author{
Pascal Rusch, ${ }^{\dagger, \S}$ Björn Schremmer, ${ }^{\dagger, \S}$ Christian Strelow, ${ }^{\ddagger}$ Alf Mews, ${ }^{\ddagger}$ Dirk Dorfs, ${ }^{\dagger, \S, \perp \odot() ~}$ \\ and Nadja C. Bigall $*, \dagger, \S, \perp$ (1) \\ ${ }^{\dagger}$ Institute of Physical Chemistry and Electrochemistry, Leibniz Universität Hannover, Callinstraße 3A, 30167 Hannover, Germany \\ ${ }^{\S}$ Laboratory of Nano and Quantum Engineering, Leibniz Universität Hannover, Schneiderberg 39, 30167 Hannover, Germany \\ ${ }^{\ddagger}$ Institute of Physical Chemistry, University of Hamburg, Grindelallee 117, 20146 Hamburg, Germany \\ ${ }^{\perp}$ Cluster of Excellence PhoenixD (Photonics, Optics, and Engineering - Innovation Across Disciplines), 30167 Hannover, Germany
}

Supporting Information

ABSTRACT: The influence of interparticle contact in nanoparticle-based aerogel network structures is investigated by selectively connecting or isolating the building blocks inside of the network, thereby coupling and decoupling them in regards to their optical and electronic properties. This is achieved by tuning the synthesis sequence and exchanging the point of shell growth and the point of particle assembly, leading to two distinctly different structures as examined by electron microscopy. By thorough examination of the resulting optical properties of the generated structures, the clear correlation between nanoscopic/microscopic structure and macroscopic optical properties is demonstrated. Temperature-dependent measurements and effective mass approximation calculations support our findings.

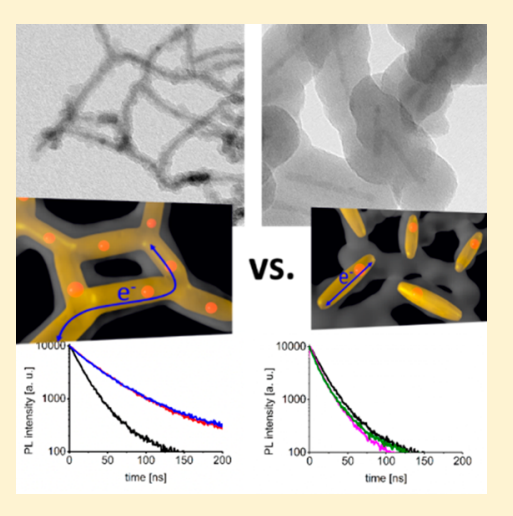

$\mathrm{O}$ ne potential method to immobilize nanoparticles and even form macroscopic solids while retaining the nanoscopic properties of colloid chemically synthesized nanocrystals is the assembly of said particles into threedimensional network structures. The network formation is achieved by controlled destabilization of a colloidal nanoparticle solution. By carefully adjusting the speed of destabilization, instead of precipitation, the particles can form an interconnected macroscopic network often called a gel. This method has been introduced for semiconductor nanoparticles ${ }^{1-3}$ and has been expanded to date with different materials, ${ }^{4-9}$ shapes, ${ }^{10-12}$ and assembly routes. ${ }^{13-15}$ Already in the first instance of such semiconductor nanoparticle-based aerogel networks, the emission of the newly generated macroscopic body was described, although at low temperature. ${ }^{1}$ It was shown that the monolithic assembly of nanoparticles in this instance shows a much higher intensity of the trap state emission compared to the initial nanoparticles. The reflectance spectroscopy still shows size quantization effects, which indicates that the nanoscopic properties of the nanoparticle building blocks can be transferred onto macroscopic bodies with these techniques. ${ }^{1}$ By using core-shell particles, the trap state emission could be avoided and the fluorescence of the nanoparticle building blocks could be completely carried over to a macroscopic solid. ${ }^{16} \mathrm{CdSe} / \mathrm{CdS}$ dot-in-rod nanostructures have already been investigated extensively regarding their optical properties and the underlying electronic processes. ${ }^{17-23}$ Building further on this structure, our group was later able to show that the assembly of nanoparticle building blocks into a network structure can actually not only be performed while retaining the optical properties of the particles but also can be used to introduce new optical properties into the final structure. ${ }^{24}$ In the work reported, $\mathrm{CdSe} / \mathrm{CdS}$ nanoparticles were used as pseudotype-II building blocks, and it could be shown that networks consisting of these building blocks have drastically increased fluorescence lifetimes. This was attributed to the excited electron being able to travel within the network of interconnected building blocks while the hole is localized in the CdSe cores of individual building blocks. ${ }^{24}$ Similarly, an influence of the aspect ratio of the nanorod (NR) building blocks on their optical properties could be shown. ${ }^{25}$ Recently, further studies have been undertaken using spectroelectrochemical measurements to understand the unique optical properties of semiconductor nanoparticle gels and suggest their applicability as sensors. ${ }^{26,27}$

In the present work, we aim to employ our recently introduced method of post-gelation modification ${ }^{28}$ to build up nanoparticle-based network structures in two different sequences with the aim of selectively coupling and decoupling the individual building blocks, as illustrated in Figure 1. The silica encapsulation of singular dispersed nanoparticles has already been described. ${ }^{29}$ A thin silica shell was grown according to this route, and such insulated nanoparticles were subsequently embedded into a silica gel monolith. The

Received: September 13, 2019

Accepted: November 5, 2019

Published: November 11, 2019 


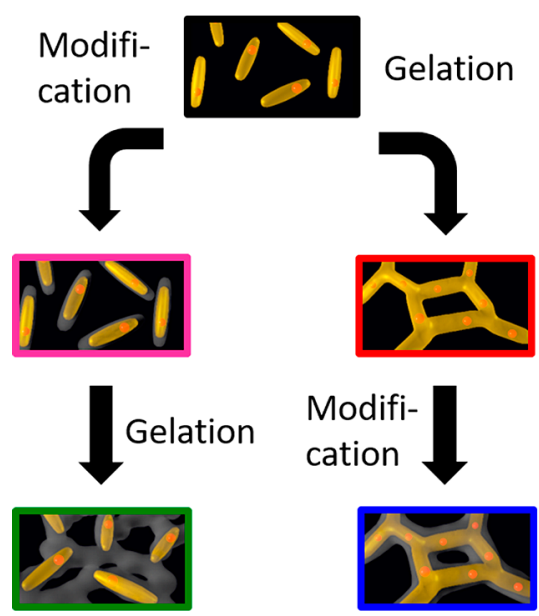

Figure 1. Schematic depiction of the two different synthesis sequences employed. Starting from (top) CdSe/CdS NR building blocks upon (right side) assembling into the network structure first and then modification by silica shell growth or (left side) first modification of the individual particles with a silica shell and then incorporation of them into a network, as illustrated by computer graphics. silica shell essentially insulates the particles and prevents direct contact between the semiconductor NRs in the macroscopic monolith. In parallel, the same $\mathrm{CdSe} / \mathrm{CdS}$ dot-in-rod nanoparticles were assembled first into an interconnected network structure. This network was afterward surrounded with a silica coating while leaving the interparticle connection intact. With this procedure, we have been able to generate a nanoparticlebased network with strongly enhanced mechanical stability, ${ }^{28}$ but in this work, we aimed to employ it to synthesize two comparable solid macroscopic porous systems (solvogels and, after supercritical drying, aerogels), one with and one without crystal-to-crystal contact between the semiconductor particles, allowing us to further investigate the influence of the interparticle contact on the optoelectronic properties.

The optical spectroscopy of the pristine CdSe/CdS NRs (Figure S2) in colloidal solution (which are the building blocks for the aerogels) in organic solution shows the characteristics of this nanoparticle structure. The absorption of the cadmium selenide core is visible as a small band at $570 \mathrm{~nm}$, while the cadmium sulfide shell is responsible for the strong absorption band at $468 \mathrm{~nm} .{ }^{30}$ Due to the electronic structure of the NRs, the hole is trapped in the CdSe core after excitation. This leads to a strong fluorescence emission at $585 \mathrm{~nm}$, slightly bathochromically shifted compared to the absorption of the core. The phase transfer ${ }^{31,32}$ to aqueous solution only
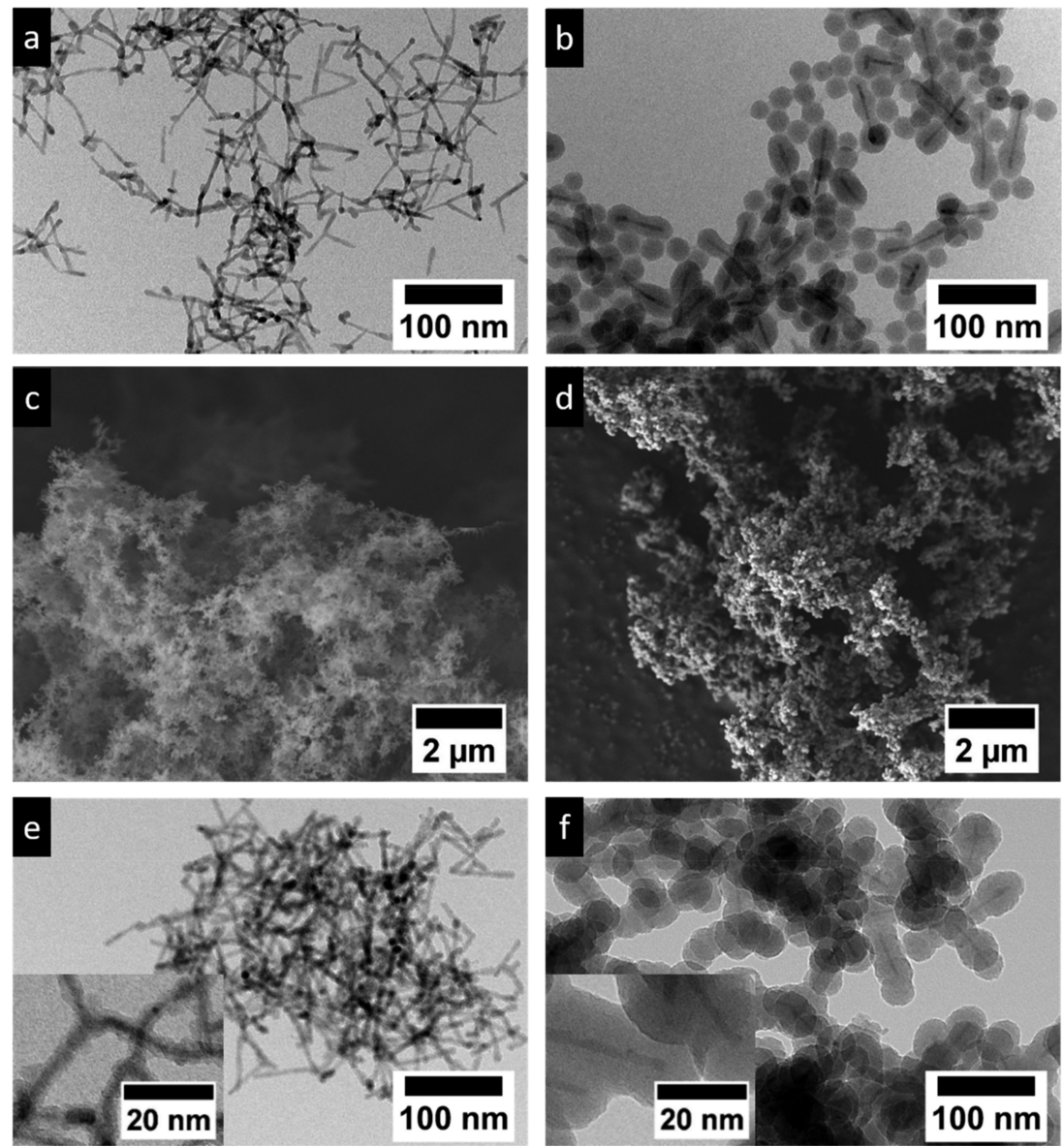

Figure 2. Electron microscopic images of the investigated structures comparing synthesis sequences starting with the gel network assembly (a,c,e) or starting with the particle modification $(\mathrm{b}, \mathrm{d}, \mathrm{f})$. (a) Initial network of interconnected CdSe/CdS NRs before shell growth in TEM; (b) silicamodified CdSe/CdS NRs in TEM; network of CdSe/CdS NRs modified with silica after network formation (c) in SEM and (e) in TEM with the inset at higher magnification; and gel network generated from silica-modified CdSe/CdS NRs (d) in SEM and (f) in TEM, with the inset at higher magnification. 


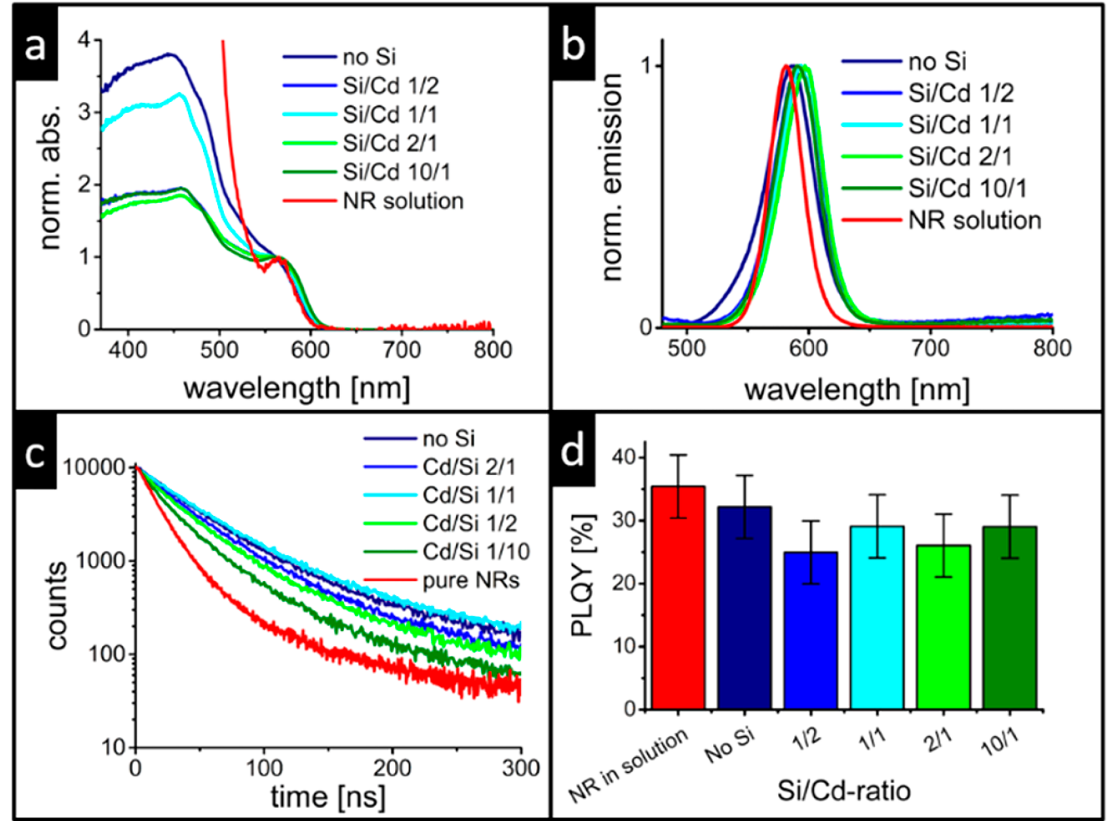

Figure 3. Optical properties of silica-modified CdSe/CdS NR aerogels: (a) absorption spectra, (b) emission spectra, (c) fluorescence lifetime decay, and (d) PLQYs of aerogels modified with different amounts of TEOS. As can be derived from the four panels, the silica shell modification does not significantly alter the optical properties (in absorption, emission, PL lifetime, and quantum yield) in comparison to the nonmodified $\mathrm{CdSe} / \mathrm{CdS} \mathrm{NR}$ aerogels.

marginally influences the absorption of the NRs; the position of the emission maximum does not change. However, the photoluminescence quantum yield (PLQY) is reduced slightly from 35 to $27 \%$. The fluorescence lifetime also decreases from $18 \mathrm{~ns}$ in the organic solution to $16 \mathrm{~ns}$ in the aqueous solution (Figure S4a,b). These NRs are assembled by controlled destabilization by hydrogen peroxide into porous network structures, as can be seen in Figure 2a. The aerogels prepared this way show very similar optical features (Figure 3 ). In the nonmodified aerogels, the emission is visible at $588 \mathrm{~nm}$. The absorption of the CdSe core can be seen at $563 \mathrm{~nm}$, while the absorption of the CdS shell appears as a broad band at around $500 \mathrm{~nm}$. Especially for the interpretation of the absorption spectra, it is important to keep the state of the sample in mind. Because of the high influence of scattering compared to a colloidal nanoparticle solution, the gel is measured using an integrating sphere. Also, the concentration of the nanoparticles is much higher than one would use for conventional nanoparticle spectroscopy in solution. This leads to the much larger prominence of the signal from the cadmium selenide core in the spectra, which is only barely visible in the spectrum of the colloidal solution. It also causes the spectra to reach saturation in the area of the cadmium sulfide absorption band. For light of energies higher than a certain value or below a certain wavelength, the gels simply become optically dense materials in which all incoming light is absorbed and the higher-energy features of the spectrum are not measurable. The findings discussed above are consistent with our previous data of such types of aerogels; ${ }^{24}$ therefore, these samples can be employed as references for our new materials, as will be discussed in the next paragraph.

In a second step, the networks are modified with a continuous silica shell, as can be seen in TEM (Figure 2e); see the Experimental Methods section for details. The porous structure is still present after this modification, as can be seen in SEM images (Figure 2c) and based on the core network of connected NRs. For silica-modified gels, the optical properties are almost identical compared to the unmodified gels, as shown in Figure 3 and discussed above. The emission wavelength is slightly shifted toward longer wavelengths from 590 to $597 \mathrm{~nm}$. This could simply be due to the change in dielectric surrounding and minor inhomogeneities during the shell growth process, but as no clear relation to the amount of silica used or other variables in the synthesis procedure is visible, the exact reason is unclear. The absorption features are also similar to the absorption of the CdSe cores around $560 \mathrm{~nm}$ and the CdS absorption below $500 \mathrm{~nm}$. The optical density of absorption saturation changes, which we attribute to a variation of illuminated sample areas (which is technically unavoidable due to inhomogeneities of the monolithic samples). The PLQY of all gels-modified and unmodified-is similar. The PLQY of the pristine NRs in toluene is measured to be $35 \%$; the PLQY of an unmodified aerogel is very close to this at $32 \%$ and a bit higher than the PLQY of the water transferred NRs (27\%). Shell-modified aerogels show PLQYs of 26-29\% (Figure 2d). Within the uncertainty (5\%, determined by reproducibility tests with the reference dye rhodamine $6 \mathrm{G}$ ) estimated for these measurements, these differences are minimal. The loss of PLQY by the silica shell modification can thus be seen as insignificant. The fluorescence lifetime of the aerogels is especially interesting as the connection of the NRs with each other is a key factor in the fluorescence decay. In an earlier work of our group, a strongly increased fluorescence lifetime for CdSe/CdS NR aerogels compared to the NRs in solution was shown and attributed to the crystal-to-crystal type connection of the rods. We explained this effect with a higher delocalization of the electron after excitation, resulting in less overlap between electron and hole wave functions and therefore longer PL decay times. ${ }^{24}$ The lifetime increase can therefore be seen as indication for an electronic coupling between interconnected nanoparticles in the aerogel networks. In fact, in the present work, we also 


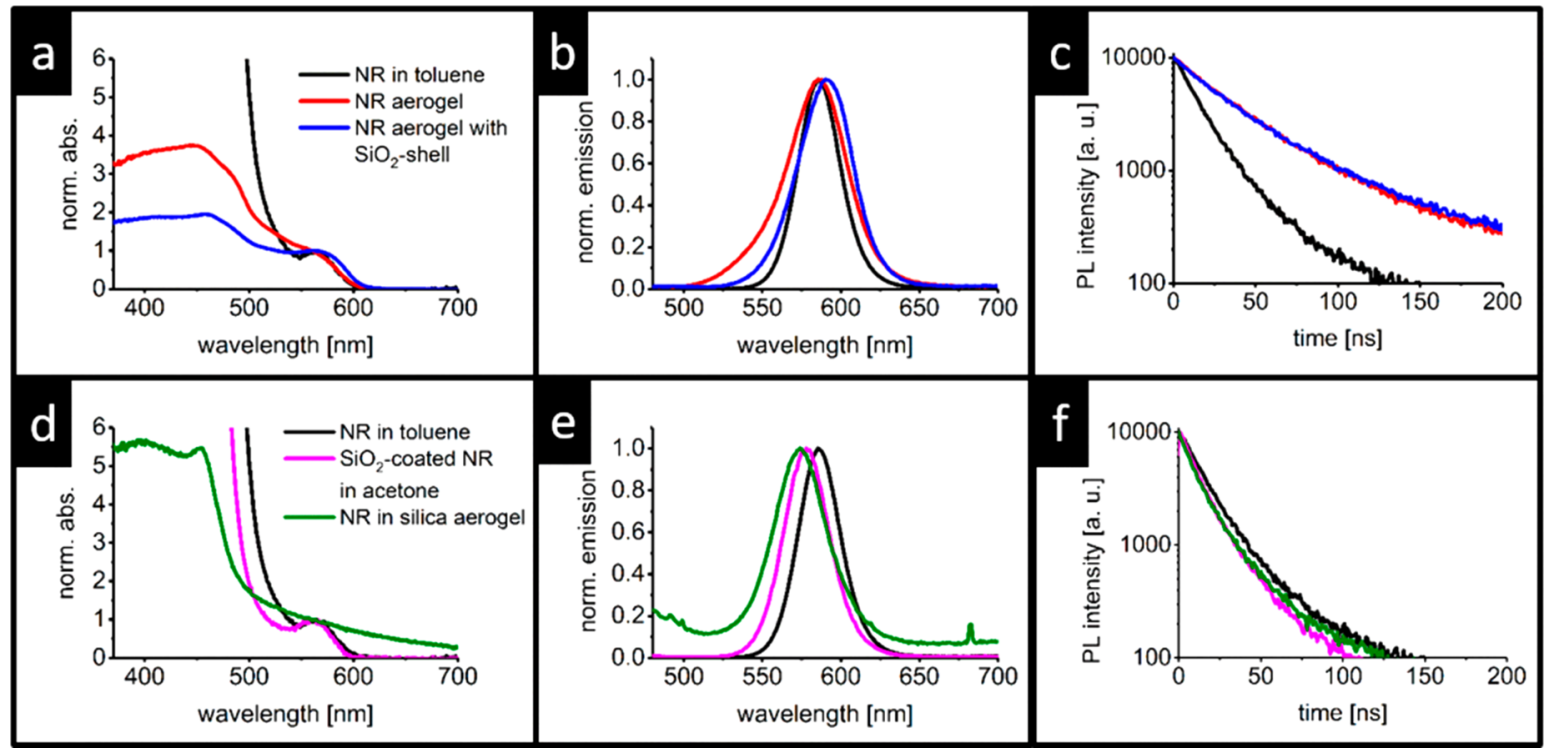

Figure 4. Differences in optical properties depending on the synthesis sequence: (top row) network formation first, (bottom row) silica modification first. (a,d) Absorption spectra normalized to the first excitonic transition of the CdSe core, (b,e) emission spectra, and (c,f) fluorescence decay measurements. Colors of the graphs also correspond to the borders used in Figure 1.

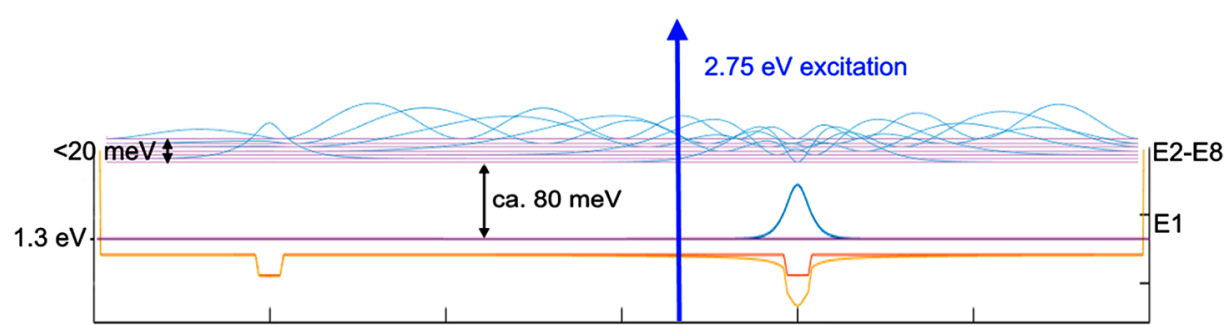

Figure 5. Wave functions of the first eight excited states in two connected CdSe/CdS NRs calculated by EMA with the hole fixed to the right side core. The external potential is shown in orange with the overall potential including coulomb interaction in yellow and the generated wave functions in blue.

observe increased fluorescence lifetimes for all aerogels-with and without a silica shell-compared to the lifetime of the NRs in solution. The fluorescence lifetime of unmodified $\mathrm{CdSe} / \mathrm{CdS} \mathrm{NR}$ aerogels is $40 \mathrm{~ns}$, which is in accordance with our previous findings. ${ }^{24}$ For modified aerogels, the lifetimes are in the range of 33-41 ns (Figures 2c and S3). This means that also the drastic lifetime increase is present for all $\mathrm{Si} / \mathrm{Cd}$ ratios investigated for silica shell-modified aerogels, which is comparable to that of unmodified aerogels. Hence, we conclude that also in the silica shell-modified aerogels the electron seems to be able to travel within the network while the hole is located at the CdSe cores. If this explanation for the increase in fluorescence lifetime is to be believed, a network containing individual isolated particles-e.g., by the growth of an insulating silica shell before network formation-should show very much different behavior regarding the fluorescence lifetimes. In this instance, fluorescence lifetimes should not increase between a particle dispersion and the network

To investigate whether the sequence of synthesis steps (namely, network formation and silica coating) influence the physical properties of the resulting nanocrystal aerogels in terms of fluorescence lifetime, the same NRs as those used for the production of the described modified gels were now first surrounded with silica shells according to literature procedures $^{29}$ (see the Experimental Methods section and Figure 2b) and subsequently gelated in the presence of further TEOS.
This resulted in a silica matrix with singular embedded NRs, which were not in direct contact with each other and should therefore have been decoupled, i.e., they should not have shown increased PL decay times (see also Figure S6 in the Supporting Information). These single NRs in a silica matrix can be seen in the TEM (Figure 2f). The larger structure of these silica networks can be seen in SEM (Figure 2d) and is now defined by the silica as opposed to the networks made up of connected NRs, which are defined by this NR core network (see also Figure 2 for comparison). The different structures are illustrated schematically in Figure 1. The silica aerogel is essentially utilized as an electronically isolating spacer between the NRs. The emission and absorption spectra of these silicacoated NRs in solution and in the silica gel only differ insignificantly from each other (Figure $4 a-e$ ). Also, no significant spectral difference of such gels and particles from the pristine NRs (before silica growth) or from the unmodified and modified aerogels is observed. The lifetime measurements confirm our initial expectation. While the aerogels produced from $\mathrm{CdSe} / \mathrm{CdS} \mathrm{NRs}$ as well as their post-gelation-modified counterparts show distinct ultralong lifetimes of ca. $40 \mathrm{~ns}$, the aerogels with embedded NRs, which are each separated from one other by a thin silica shell, exhibit much shorter lifetimes of 13-14 ns (which are comparable to those of the initial NRs in organic solution) (Figure $4 c, f)$. These observations further support our previous assumption of the influence of 
interparticle contact on the fluorescence lifetime in NR gels ${ }^{24}$ and show that this contact between the individual NRs is still present after post-gelation modification.

To understand the electronic processes underlying the observed fluorescence behavior, theoretical calculations based on the effective mass approximation were performed to simulate the excited electron wave functions of a network structure of crystal connected particles. Figure 5 shows the results for the eight lowest excited electron wave functions of two merged CdSe/CdS NRs that should represent the smallest subunit in the network exhibiting the properties of the whole structure. Due to its low effective mass and the high valence band discontinuity of $\mathrm{CdS}$ and $\mathrm{CdSe}$, the hole always localizes inside of one of the two CdSe cores (not shown in Figure 5). The first excited electron state (E1 in Figure 5) also strongly localizes at the position of the hole due to the combination of the attractive potential formed by conduction band discontinuity and the coulomb interaction with the localized whole. The properties of this state do not differ from those of the corresponding state in an isolated particle and thus do not explain the observed increase in PL lifetime. The second excited electron state (E2) is also localized at the second CdSe core not occupied by the hole. Due to the strongly reduced coulomb interaction with the hole, it is lifted in energy by about $80 \mathrm{meV}$. However, the next higher excited electron states (E3-E8; see Figure 5 and additionally Figure S7 in the Supporting Infomation for the individual wave functions) exhibit an increasing delocalization over the merged CdSe/ CdS NR system with increasing energy. The wave functions of these higher electron states indeed have a smaller overlap with the hole wave function. We thus attribute these higher electron states to be responsible for the observed fluorescence kinetics. Naturally, the question arises why the electron and the hole do not thermalize into the lowest- energy state that does not show a difference from the individual isolated $\mathrm{CdSe} / \mathrm{CdS} \mathrm{NR}$.

We explain this contradiction as follows: the excitation with $450 \mathrm{~nm}$ light used in the measurements creates pairs of hot electrons and holes that occupy higher excited states. Subsequently, the electron and hole separate and thermalize independently, leading to either (i) the occupation of states in different cores or (ii) direct occupation of a delocalized higher electron state. The first case (i) would lead to zero overlap of the electron and hole wave functions and would not contribute to the measured fluorescence. However, as can be observed in Figure 5, the second excited electron state (E2) that is separated from the hole has an energy very close to a bunch of higher electron states (labeled E3-E8 in Figure 5), which are all within an energy range of $10-20 \mathrm{meV}$ of each other. Thus, thermal activation of the separated electron into the delocalized states or even a thermal mixing of all higher excited electron states (E2-E8) is reasonable. This also includes the above-mentioned second case (ii), direct thermalization into a delocalized (thermally mixed) higher electron state. The wave functions of the thermally mixed higher excited electron states should in general have a reduced overlap with the localized hole and should thus lead to a reduced fluorescence lifetime and/or even to a suppression of the thermalization into the lowest excited electron state (E1).

Following this explanation, the influence of the states showing considerable delocalization (labeled E5-E8 in Figure S7) should decrease with lower temperature, leading to higher wave function overlap and shorter PL lifetimes. To confirm this temperature-dependent lifetime, measurements have been carried out as well. These indeed show a decrease in PL lifetime with lower temperatures (see Figure S8 in the Supporting Information) contrary to classical theory, which assumes no influence of temperature on the radiative process and a slowing down of the non-radiative processes leading to increased PL lifetimes. Because we observe the opposite effect within our measurements, a different factor influencing the lifetimes has to exist, and our above-mentioned thermal mixing of higher excited states becomes reasonable.

In this work, the possibility to couple and decouple the nanoparticle building blocks when assembling them into a network structure is shown. This is achieved by switching the synthesis sequence during the generation of the network, i.e., either first generating a nanoparticle-based network and subsequently surrounding this network with a silica shell or first isolating the individual particles with silica shells and subsequently embedding them into a silica-based network. The pronounced effect of the interparticle contact on the optical properties in the first case could be illustrated by photoluminescence spectroscopy and further detailed with theoretical calculations. When a contact between the particles is established before silica shell growth, the ultralong lifetimes typical for these $\mathrm{CdSe} / \mathrm{CdS}$ network structures are visible independently of the presence of a silica shell. If the direct contact between the semiconductor NRs is instead inhibited by silica coating of individual particles, an increase in lifetime can not be observed. This supports the underlying theory that electrons can travel within such nanocrystal networks. This model was supported by effective mass approximation simulations in combination with temperature-dependent lifetime measurements. Our work furthermore shows that the sequence of synthetic steps in nanochemistry is of utmost importance for controlling the optoelectronic properties of such elaborate materials.

\section{EXPERIMENTAL METHODS}

The preparation of the $\mathrm{CdSe} / \mathrm{CdS}$ building blocks has been described in earlier works ${ }^{30}$ as well as their phase transfer, ${ }^{31,32}$ assembly, ${ }^{24}$ silica modification of dispersed particles, ${ }^{29}$ and modification of networks. ${ }^{28}$ It is additionally described briefly in the Supporting Information.

Optical Characterization. Absorption measurements were performed in an Agilent Cary 5000 spectrophotometer in $3 \mathrm{~mL}$ quartz cuvettes with a $10 \mathrm{~mm}$ path length. For the measurement of aerogels, a DRA-2500 integrating sphere was connected to the spectrophotometer, and the sample was mounted in reflection position. Photoluminescence measurements were carried out in a Horiba Fluoromax- 4 spectrometer for emission and lifetime measurements. The lifetime was measured by time-correlated single-photon counting (TCSPC) using a NanoLED with a $455 \mathrm{~nm}$ wavelength as the excitation source. Each lifetime measurement was continued until the peak value reached 10000 counts. The measurement of PLQY was done by absolute measurements using a Horiba Dual-FL with a quanta- $\phi$ integrating sphere with an excitation wavelength of $500 \mathrm{~nm}$. Therefore, the intensity of the excitation was adjusted to be below the detector limit, and emission spectra were measured over the range of 300$800 \mathrm{~nm}$ for an empty integration sphere and again with the sample. The difference between the integrated area under the excitation peak in these two measurements is equivalent to the total number of photons absorbed, while the difference in the integrated area under the emission peak is equivalent to the 
number of photons emitted. By forming the quotient of these areas, the quantum yield was calculated. The error of the quantum yield measurement was estimated by multiple measurements of rhodamine 6G over 14 days to be about absolute 5\%. Temperature dependent-fluorescence measurements were carried out using an Edinburgh FLS 1000 spectrometer equipped with an Oxford Instruments OptistatCF cryostat cooled with liquid helium. For this measurement, the aerogel samples were put inside of an open quartz cuvette with a $2 \mathrm{~mm}$ path length, and the sample space was flushed with helium. For time-correlated measurements, the samples were excited by an Edinburgh EPL pulsed laser with a $445.1 \mathrm{~nm}$ wavelength.

Electron Microscopy. TEM samples of nanoparticles in solution were prepared by drop-casting diluted solutions in chloroform onto a carbon-coated copper grid (300 mesh) by Quantifoil. For sample preparation of aerogels, the mentioned grids were pulled along an aerogel sample, thereby keeping small parts of gel stuck to the grid. The measurements were carried out in an FEI Tecnai G2 F20 transmission electron microscope with a field emission gun operated at $200 \mathrm{kV}$.

SEM samples were prepared by dropping fragments of the gel onto an adhesive carbon film and shaking off loosely attached parts. A JEOL JSM 6700F field emission scanning electron microscope with a $2 \mathrm{kV}$ acceleration voltage and a secondary electron detector at a working distance of $8 \mathrm{~mm}$ was used to measure the samples.

Theoretical Calculations. The simulations were done by selfconsistently solving the Schrödinger equation for one electron and one hole on a three-dimensional spatial grid in a singleband effective mass approximation. The potential was composed of the external potential formed by the band alignments and the Coulomb potential generated for the electron by the hole and for the hole by the electron. Electron and hole wave functions and eigenenergies were calculated iteratively until convergence was achieved. The band gaps of $\mathrm{CdS}$ and $\mathrm{CdSe}$ were set to 2.41 and $1.74 \mathrm{eV}$, respectively, and the conduction band offset was set to $150 \mathrm{meV}$.

\section{ASSOCIATED CONTENT}

\section{S Supporting Information}

The Supporting Information is available free of charge at https://pubs.acs.org/doi/10.1021/acs.jpclett.9b02695.

Additional characterization of the nanocrystal building blocks and networks, further fluorescence lifetime measurements and evaluation including temperaturedependent measurements, synthesis procedures, and the band structure of the discussed materials, and further results of theoretical calculations (PDF)

\section{AUTHOR INFORMATION}

\section{Corresponding Author}

*E-mail: nadja.bigall@pci.uni-hannover.de. Address: Institute of Physical Chemistry and Electrochemistry, Callinstraße 3A, 30167 Hannover, Germany.

\section{ORCID $\odot$}

Alf Mews: 0000-0001-5739-8820

Dirk Dorfs: 0000-0001-6175-4891

Nadja C. Bigall: 0000-0003-0171-1106

Notes

The authors declare no competing financial interest.

\section{ACKNOWLEDGMENTS}

The authors would like to thank the Laboratory of Nano and Quantum Engineering for the use of the TEM, as well as J. Caro and A. Feldhoff for SEM. The project leading to these results received funding from the European Research Council (ERC) under the European Union's Horizon 2020 research and innovation programme (Grant Agreement No. 714429). N.C.B. furthermore acknowledges funding from the German Federal Ministry of Education and Research (BMBF) within the framework of NanoMatFutur (Support Code 03X5525). D.D. acknowledges funding by the DFG (Research Grant $1580 / 5-1)$. The project has in part been funded by the Deutsche Forschungsgemeinschaft (DFG, German Research Foundation) under Germany's Excellence Strategy within the Cluster of Excellence PhoenixD (EXC 2122, Project ID 390833453).

\section{REFERENCES}

(1) Mohanan, J. L.; Arachchige, I. U.; Brock, S. L. Porous Semiconductor Chalcogenide Aerogels. Science 2005, 307, 397-400.

(2) Gacoin, T.; Malier, L.; Boilot, J. P. New Transparent Chalcogenide Materials Using a Sol-Gel Process. Chem. Mater. 1997, 9 (7), 1502-1504.

(3) Gacoin, T.; Lahlil, K.; Larregaray, P.; Boilot, J. P. Transformation of CdS Colloids: Sols, Gels, and Precipitates. J. Phys. Chem. B 2001, 105 (42), 10228-10235.

(4) Ganguly, S.; Brock, S. L. Toward Nanostructured Thermoelectrics: Synthesis and Characterization of Lead Telluride Gels and Aerogels. J. Mater. Chem. 2011, 21 (24), 8800.

(5) Hitihami-Mudiyanselage, A.; Senevirathne, K.; Brock, S. L. Assembly of Phosphide Nanocrystals into Porous Networks: Formation of InP Gels and Aerogels. ACS Nano 2013, 7 (2), $1163-1170$.

(6) Kalebaila, K. K.; Georgiev, D. G.; Brock, S. L. Synthesis and Characterization of Germanium Sulfide Aerogels. J. Non-Cryst. Solids 2006, 352 (3), 232-240.

(7) Bigall, N. C.; Herrmann, A.-K.; Vogel, M.; Rose, M.; Simon, P.; Carrillo-Cabrera, W.; Dorfs, D.; Kaskel, S.; Gaponik, N.; Eychmüller, A. Hydrogels and Aerogels from Noble Metal Nanoparticles. Angew. Chem., Int. Ed. 2009, 48 (51), 9731-9734.

(8) Gaponik, N.; Wolf, A.; Marx, R.; Lesnyak, V.; Schilling, K.; Eychmüller, A. Three-Dimensional Self-Assembly of Thiol-Capped CdTe Nanocrystals: Gels and Aerogels as Building Blocks for Nanotechnology. Adv. Mater. 2008, 20 (22), 4257-4262.

(9) Yao, Q.; Arachchige, I. U.; Brock, S. L. Expanding the Repertoire of Chalcogenide Nanocrystal Networks: $\mathrm{Ag}_{2}$ Se Gels and Aerogels by Cation Exchange Reactions. J. Am. Chem. Soc. 2009, 131 (8), 28002801.

(10) Yu, H.; Brock, S. L. Effects of Nanoparticle Shape on the Morphology and Properties of Porous CdSe Assemblies (Aerogels). ACS Nano 2008, 2 (8), 1563-1570.

(11) Naskar, S.; Miethe, J. F.; Sanchez-Paradinas, S.; Schmidt, N.; Kanthasamy, K.; Behrens, P.; Pfnür, H.; Bigall, N. C. Photoluminescent Aerogels from Quantum Wells. Chem. Mater. 2016, 28 (7), 2089-2099.

(12) Naskar, S.; Freytag, A.; Deutsch, J.; Wendt, N.; Behrens, P.; Köckritz, A.; Bigall, N. C. Porous Aerogels from Shape-Controlled Metal Nanoparticles Directly from Nonpolar Colloidal Solution. Chem. Mater. 2017, 29 (21), 9208-9217.

(13) Sayevich, V.; Cai, B.; Benad, A.; Haubold, D.; Sonntag, L.; Gaponik, N.; Lesnyak, V.; Eychmüller, A. 3D Assembly of AllInorganic Colloidal Nanocrystals into Gels and Aerogels. Angew. Chem., Int. Ed. 2016, 55 (21), 6334-6338.

(14) Freytag, A.; Sánchez-Paradinas, S.; Naskar, S.; Wendt, N.; Colombo, M.; Pugliese, G.; Poppe, J.; Demirci, C.; Kretschmer, I.; Bahnemann, D. W.; et al. Versatile Aerogel Fabrication by Freezing 
and Subsequent Freeze-Drying of Colloidal Nanoparticle Solutions. Angew. Chem., Int. Ed. 2016, 55 (3), 1200-1203.

(15) Ranmohotti, K. G. S.; Gao, X.; Arachchige, I. U. Salt-Mediated Self-Assembly of Metal Nanoshells into Monolithic Aerogel Frameworks. Chem. Mater. 2013, 25 (17), 3528-3534.

(16) Arachchige, I. U.; Brock, S. L. Highly Luminescent QuantumDot Monoliths. J. Am. Chem. Soc. 2007, 129 (7), 1840-1841.

(17) Steiner, D.; Dorfs, D.; Banin, U.; Della Sala, F.; Manna, L.; Millo, O. Determination of Band Offsets in Heterostructured Colloidal Nanorods Using Scanning Tunneling Spectroscopy. Nano Lett. 2008, 8 (9), 2954-2958.

(18) Talapin, D. V.; Koeppe, R.; Götzinger, S.; Kornowski, A.; Lupton, J. M.; Rogach, A. L.; Benson, O.; Feldmann, J.; Weller, H. Highly Emissive Colloidal CdSe/CdS Heterostructures of Mixed Dimensionality. Nano Lett. 2003, 3 (12), 1677-1681.

(19) Kunneman, L. T.; Zanella, M.; Manna, L.; Siebbeles, L. D. A.; Schins, J. M. Mobility and Spatial Distribution of Photoexcited Electrons in CdSe/CdS Nanorods. J. Phys. Chem. C 2013, 117 (6), $3146-3151$.

(20) Saba, M.; Minniberger, S.; Quochi, F.; Roither, J.; Marceddu, M.; Gocalinska, A.; Kovalenko, M. V.; Talapin, D. V.; Heiss, W.; Mura, A.; et al. Exciton-Exciton Interaction and Optical Gain in Colloidal CdSe/CdS Dot/Rod Nanocrystals. Adv. Mater. 2009, 21 (48), 4942-4946.

(21) Kraus, R. M.; Lagoudakis, P. G.; Rogach, A. L.; Talapin, D. V.; Weller, H.; Lupton, J. M.; Feldmann, J. Room-Temperature Exciton Storage in Elongated Semiconductor Nanocrystals. Phys. Rev. Lett. 2007, 98 (1), 017401.

(22) Vezzoli, S.; Manceau, M.; Leménager, G.; Glorieux, Q.; Giacobino, E.; Carbone, L.; De Vittorio, M.; Bramati, A. Exciton Fine Structure of $\mathrm{CdSe} / \mathrm{CdS}$ Nanocrystals Determined by Polarization Microscopy at Room Temperature. ACS Nano 2015, 9 (8), 79928003.

(23) Persano, A.; De Giorgi, M.; Fiore, A.; Cingolani, R.; Manna, L.; Cola, A.; Krahne, R. Photoconduction Properties in Aligned Assemblies of Colloidal CdSe/CdS Nanorods. ACS Nano 2010, 4 (3), 1646-1652.

(24) Sanchez-Paradinas, S.; Dorfs, D.; Friebe, S.; Freytag, A.; Wolf, A.; Bigall, N. C. Aerogels from CdSe/CdS Nanorods with Ultra-Long Exciton Lifetimes and High Fluorescence Quantum Yields. Adv. Mater. 2015, 27 (40), 6152-6156.

(25) Geißler, D.; Würth, C.; Wolter, C.; Weller, H.; Resch-Genger, $\mathrm{U}$. Excitation Wavelength Dependence of the Photoluminescence Quantum Yield and Decay Behavior of CdSe/CdS Quantum Dot/ Quantum Rods with Different Aspect Ratios. Phys. Chem. Chem. Phys. 2017, 19 (19), 12509-12516.

(26) Lübkemann, F.; Miethe, J. F.; Steinbach, F.; Rusch, P.; Schlosser, A.; Zámbó, D.; Heinemeyer, T.; Natke, D.; Zok, D.; Dorfs, D.; et al. Patterning of Nanoparticle-Based Aerogels and Xerogels by Inkjet Printing. Small 2019, 15, 1902186.

(27) Schlosser, A.; Meyer, L. C.; Lübkemann, F.; Miethe, J. F.; Bigall, N. C. Nanoplatelet Cryoaerogels with Potential Application in Photoelectrochemical Sensing. Phys. Chem. Chem. Phys. 2019, 21 (18), 9002-9012.

(28) Rusch, P.; Niemeyer, F.; Pluta, D.; Schremmer, B.; Lübkemann, F.; Rosebrock, M.; Schäfer, M.; Jahns, M.; Behrens, P.; Bigall, N. C. Versatile Route to Core-shell Reinforced Network Nanostructures. Nanoscale 2019, 11 (32), 15270-15278.

(29) Tang, X.; Kröger, E.; Nielsen, A.; Strelow, C.; Mews, A.; Kipp, T. Ultrathin and Highly Passivating Silica Shells for Luminescent and Water-Soluble CdSe/CdS Nanorods. Langmuir 2017, 33 (21), 52535260.

(30) Carbone, L.; Nobile, C.; De Giorgi, M.; Sala, F. D.; Morello, G.; Pompa, P.; Hytch, M.; Snoeck, E.; Fiore, A.; Franchini, I. R.; et al. Synthesis and Micrometer-Scale Assembly of Colloidal CdSe/CdS Nanorods Prepared by a Seeded Growth Approach. Nano Lett. 2007, 7 (10), 2942-2950.
(31) Kodanek, T.; Banbela, H. M.; Naskar, S.; Adel, P.; Bigall, N. C.; Dorfs, D. Phase Transfer of 1- and 2-Dimensional Cd-Based Nanocrystals. Nanoscale 2015, 7 (45), 19300-19309.

(32) Bagaria, H. G.; Ada, E. T.; Shamsuzzoha, M.; Nikles, D. E.; Johnson, D. T. Understanding Mercapto Ligand Exchange on the Surface of FePt Nanoparticles. Langmuir 2006, 22 (18), 7732-7737. 